\title{
Lyoprotective Effect of Alkyl Sulfobetaines for Freeze-drying 1,2-Dipalmitoyl-sn-glycero-3- phosphocholine Liposomes
}

\author{
Tatsuo Aikawa ${ }^{1}$, Kanta Sato ${ }^{1}$, Hiroki Okado ${ }^{1}$, Yukako Takahashi ${ }^{1}$, Takeshi Kondo ${ }^{1,2}$ and \\ Makoto Yuasa ${ }^{1,2, *}$ \\ ${ }^{1}$ Department of Pure and Applied Chemistry, Faculty of Science and Technology, Tokyo University of Science, 2641 Yamazaki, Noda, Chiba 278- \\ 8510, JAPAN \\ ${ }^{2}$ Research Institute for Science and Technology, Tokyo University of Science, 2641 Yamazaki, Noda, Chiba 278-8510, JAPAN
}

\begin{abstract}
A liposome is a molecular assembly in the form of a vesicle comprised of a phospholipid bilayer. Liposomes can be used as molecular containers in various fields such as pharmaceutical, cosmetic, and food industries. It is difficult to maintain the original structure of liposomes in an aqueous medium. Phospholipids, which are components of liposomes, are susceptible to hydrolysis, which causes disruption of the liposomal structure and dysfunction of the molecular container. In this context, freeze-drying liposomes is a preferable method to improve the shelf life of liposomes. However, when freeze-drying liposomes, a lyoprotective agent is required to preserve their original structure. In this study, we investigate whether alkyl sulfobetaines $\left(\mathrm{SB}_{n}, n\right.$ : number of carbons in the alkyl chain, $\left.n=1-18\right)$ can be used as lyoprotectants for 1,2-dipalmitoyl-sn-glycero-3-phosphocholine (DPPC) liposomes. The results indicated that the length of the alkyl chain of the $\mathbf{S B}_{n}$ was an important factor to prevent liposome disruption during the freeze-drying and subsequent rehydration processes. The use of $\mathrm{SB}_{n}$ with an alkyl chain of intermediate length $(n=6-10)$ could prevent liposome disruption and remarkably reduce the gel-to-liquid crystal phase transition temperature $\left(T_{\mathrm{m}}\right)$ of the freeze-dried liposomes. This indicates that these $\mathrm{SB}_{n}$ could intercalate in the dried bilayer and reduce intermolecular interaction between DPPC in the bilayer. The $T_{\mathrm{m}}$ reduction of the freezedried liposomes should contribute to prevention of the gel-to-liquid phase transition of the liposomes during the rehydration process, which has been known to be a main cause of liposome disruption. We expect that the results from this study will provide an insight into the influence of zwitterionic additives on freeze-dried lipid bilayers and the lyoprotective effect, which should be useful in many biochemical and biomedical fields.
\end{abstract}

Key words: liposome, 1,2-dipalmitoyl-sn-glycero-3-phosphocholine (DPPC), lyoprotection, freeze-drying, alkyl sulfobetaine

\section{Introduction}

A liposome is a vesicle composed of phospholipids, whose diameter ranges from several tens of nanometers to several micrometers. Since liposomes can encapsulate a wide variety of molecules, they may act as molecular containers, which can be used in various fields like the pharmaceutical (drug delivery system) $)^{1-6)}$, cosmetic ${ }^{7,8)}$ and food industries $^{9-11)}$. In general, liposomes can be obtained as an aqueous dispersion containing vesicular phospholipids. In the aqueous dispersion, phospholipids constituting the liposomes are sensitive to hydrolysis ${ }^{12)}$. When using phos- pholipids with an unsaturated acyl group, the lipids are sensitive to oxidation as well ${ }^{13)}$. Water and oxygen in the lipid dispersion exacerbate these undesirable reactions ${ }^{12}$. Hydrolysis and oxidation trigger disruption of the vesicular structure of the liposomes ${ }^{14)}$. Such disruptions induce fusion/aggregation of the liposomes, resulting in increment in liposome size. This causes dysfunction of the liposomes as molecular containers. For example, if the liposomes are being used as drug delivery carriers, a change in their size may deprive the liposomes of their drug accumulation function, which relies on their enhanced permeability and

\footnotetext{
*Correspondence to: Makoto Yuasa, Department of Pure and Applied Chemistry, Faculty of Science and Technology, Tokyo University of Science, 2641 Yamazaki, Noda, Chiba 278-8510, JAPAN

E-mail: yuasa@rs.noda.tus.ac.jp

Accepted June 8, 2017 (received for review April 19, 2017)

Journal of Oleo Science ISSN 1345-8957 print / ISSN 1347-3352 online

http://www.jstage.jst.go.jp/browse/jos/ http://mc.manusriptcentral.com/jjocs
} 


\section{T. Aikawa, K. Sato, H. Okado et al.}

retention effect. Leakage of encapsulated molecules from the liposomes is also induced by liposome disruption. Thus, the short shelf life of aqueous dispersions of liposomes limits their applicability as molecular containers.

In this context, lyophilization, which is also called freezedrying, has been proposed as a preferable method to remove water and oxygen contained in the liposome dispersion $^{15)}$. However, the vesicular structure of liposomes is destabilized during the freeze-drying (FD) and subsequent rehydration $(\mathrm{RH})$ processes. To prevent disruption of the liposomes during the $\mathrm{FD} / \mathrm{RH}$ processes, the use of additives called "lyoprotectants" are required. Lyoprotectants can prevent the collapse of liposome structure and leakage of entrapped molecules from the liposomes even after FD/RH of the liposomes. To date, it has been demonstrated that some types of lyoprotectants, such as amino acids ${ }^{16,17)}$, carbohydrates $^{18)}$ (e.g. glucose ${ }^{19)}$, trehalose ${ }^{20,21)}$, arbutine ${ }^{22)}$ ) and glycerol $^{23)}$ could exert a lyoprotective effect. In particular, trehalose could work effectively as a lyoprotectant.

In this study, we propose alkyl sulfobetaines $\left(\mathrm{SB}_{n}, n\right.$ : number of carbons in the alkyl chain, $n=1-18$ ) as a new class of lyoprotectants for liposomes composed of 1,2-dipalmitoyl-sn-glycero-phosphocholine (DPPC), which is a typical phospholipid. Molecular structures of $\mathrm{SB}_{n}$ and DPPC are shown in Fig. 1. The lyoprotective effect derived from trehalose is based on strong binding of trehalose to the zwitterionic headgroups of the phospholipids in the dried state. Although the molecular structure of $\mathrm{SB}_{n}$ is completely different from that of trehalose, we expect that $\mathrm{SB}_{n}$ can also act as lyoprotectants. As shown in Fig. 1, the

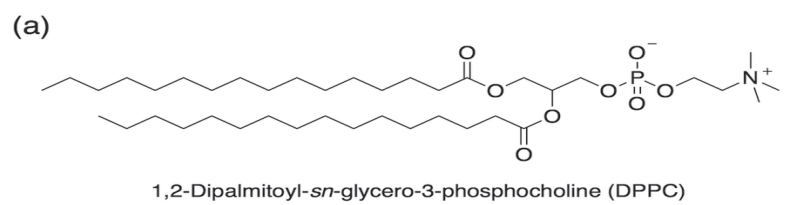

(b)

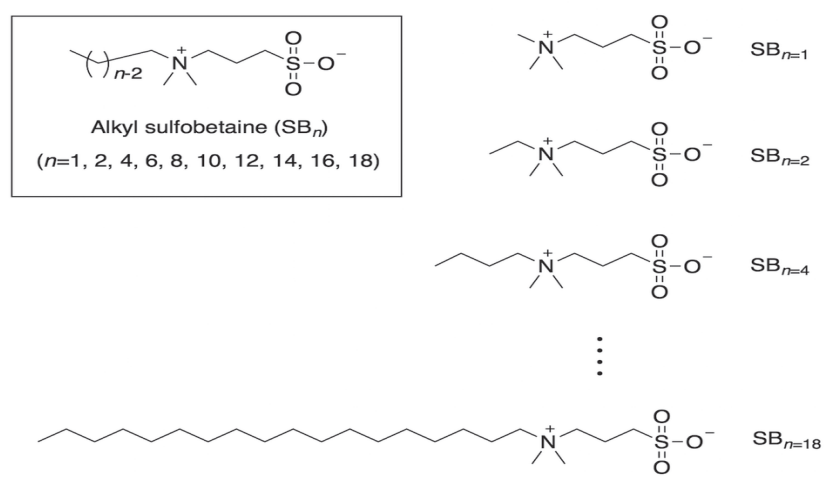

Fig. 1 Chemical structures of phospholipid and alkyl sulfobetaines $\left(\mathrm{SB}_{n}, n\right.$ : number of carbons in the alkyl chain, $n=1-18$ ) used in this study. headgroup charge in $\mathrm{SB}_{n}$ is reversely arranged with respect to the zwitterionic headgroup of DPPC. In our previous report, we revealed that there was an attractive interaction between sulfobetaine and phosphocholine moieties of 1,2diacyl glycerides ${ }^{24)}$. Considering that the lyoprotective effect is based on binding of additives to headgroups of liposomes, $\mathrm{SB}_{n}$ are expected to be lyoprotectants for DPPC liposomes because sulfobetaine can attractively interact with phosphocholine, which is the headgroup of DPPC.

In this study, we examined the applicability of $\mathrm{SB}_{n}$ as lyoprotective agents for DPPC liposomes. In order to investigate whether DPPC liposomes collapsed during FD/RH, liposome sizes were compared before and after FD/RH. In addition, to examine the integrity of the lipid bilayer of the liposomes after FD/RH, the amount of model solutes in the liposomes after FD/RH was compared to that before FD/ $\mathrm{RH}$. The effect of $\mathrm{SB}_{n}$ that were intercalated in the hydrated or freeze-dried lipid bilayer on the intermolecular interaction between DPPC was examined by differential scanning calorimetry (DSC).

\section{Materials and Method}

\subsection{Materials}

1,2-Dipalmitoyl-sn-glycero-3-phosphocholine (DPPC) was purchased from Yuka Sangyo Co. Ltd. (Tokyo, Japan). Alkyl sulfobetaines were purchased from the industries or synthesized by our group (see Supporting information). 5 (6) -Carboxyfluorescein was purchased from Sigma-Aldrich (MO, USA). All reagents were used without further purification.

\subsection{Preparation of the liposomes}

Liposomes were prepared by a conventional sonication method. Briefly, DPPC $(100 \mu \mathrm{mol})$ and SB $(25 \mu \mathrm{mol})$ were added to a round-bottom flask and dissolved in methanol. The lipids/methanol solution was slowly evaporated on a rotary evaporator under reduced pressure to form a uniformly thin lipid film on the bottom of the flask. The dried lipid film was completely hydrated with $5 \mathrm{~mL}$ of distilled water at $55^{\circ} \mathrm{C}$ (above the gel-to-liquid crystal phase transition temperature of DPPC) for 0.5 hours. The hydrated lipid film was vigorously stirred with a probe-type sonicator (UD-200, TOMY, Tokyo, Japan) at $30 \mathrm{~W}$ until the appearance of the dispersion changed from milky white to hazy transparent. The resulting dispersion was incubated for $1 \mathrm{~h}$ at $55^{\circ} \mathrm{C}$, and then filtered $(0.22 \mu \mathrm{m}$-pore $)$.

\subsection{Lyophilization and rehydration of the liposome disper- sion}

Lyophilization of the liposome dispersion was performed using a freeze dryer(FDU-1200, EYELA, Tokyo, Japan). The prepared liposome dispersion was added to a $5 \mathrm{~mL}$ 
glass vial and frozen in liquid nitrogen. The frozen sample was placed under reduced pressure $(\sim 13 \mathrm{~Pa})$ at room temperature. The lyophilized lipid cakes were rehydrated in deionized water at $55^{\circ} \mathrm{C}$ for $1 \mathrm{~h}$.

\subsection{Measurement of the size distribution}

To determine the size distribution of the liposomes before and after lyophilization, dynamic light scattering (DLS) measurements were carried out using Nicomp 380 (Particle Sizing Systems, CA, USA) equipped with a helium-neon laser $(5 \mathrm{~mW})$ operating at a wavelength of 632.8 $\mathrm{nm}$. The scattering light was collected at a $90^{\circ}$ angle at $25^{\circ} \mathrm{C}$. All sample dispersions were filtered through $0.2-\mu \mathrm{m}$ syringe filters before analysis.

\subsection{Freeze-fractured transmission electron microscopy (FF-TEM)}

The liposome dispersion was frozen rapidly in liquid nitrogen and fractured using a cold knife (FR-7000A, Hitachi High-Technologies, Tokyo, Japan). To prepare the liposome replicas, platinum vapor was exposed to the cross section of the frozen sample, and then the cross section was treated with carbon vapor to build up the replica. Liposomes on the replica were washed off with methanol and distilled water, after which the replica was transferred onto a copper grid. The liposome replica was visualized using a transmission electron microscope (H-7650, Hitachi HighTechnologies) with an acceleration voltage of $120 \mathrm{kV}$ at $-160^{\circ} \mathrm{C}$.

\subsection{Solute retention study}

To investigate the integrity of the lipid bilayer of the liposomes after $\mathrm{FD} / \mathrm{RH}$, the amount of solute entrapped in the liposomes after FD/RH was compared to that before the FD/RH. The liposomes were prepared by the same method, except for the hydration buffer. For this solute retention study, we used carboxyl fluorescein $(\mathrm{CF})$ as a model solute, which is typically used for such retention studies. The following method was used for determining the CF retention value. CF was dissolved in TES buffer $(10 \mathrm{mM}, \mathrm{pH}$ 7.58). The CF solution (10 mM in TES) was used for hydration of the lipid thin layer during liposome preparation. 4 $\mathrm{mL}$ of the liposome dispersion was divided into two aliquots (aliquot $\mathrm{A}$, aliquot $\mathrm{B}$, each volume: $2 \mathrm{~mL}$ ). To remove free $\mathrm{SB}_{n}$ and $\mathrm{CF}$ from aliquot $\mathrm{A}$, which had not been encapsulated in the liposomes following their preparation, aliquot A was passed over Sephadex G-50 (GE Healthcare Japan, Hino, Tokyo) packed in a column (15 mm $\times 113 \mathrm{~mm}$ ), and then lyophilized. In contrast, aliquot B was lyophilized immediately after liposome preparation. Lyophilized aliquots A and B were rehydrated using TES buffer. To release the entrapped CF from the liposomes into the bulk solution, the liposomes were lysed with Triton-X 100 (final concentration $=0.02 \mathrm{wt} \%$ ). The CF con- centration in the bulk solution was determined by fluorometry using FP-8000(JASCO, Tokyo, Japan). The fluorescence intensity at $515 \mathrm{~nm}(\mathrm{Ex}: 490 \mathrm{~nm})$ was measured and converted to concentration using a calibration curve.

\subsection{Differential scanning calorimetry (DSC)}

DSC measurements were carried out with DSC 1 (Mettler Toledo, Switzerland). Two different types of liposome samples, aqueous dispersions and lyophilized powders, were applied in the measurement. These samples were carefully added to aluminum pans $(40-\mu \mathrm{L}$ pan for the powders, $100-\mu \mathrm{L}$ pan for the dispersions) and then sealed with an aluminum lid. The temperature was controlled using a heating-cooling-heating cycle scan program with a scan rate of $2^{\circ} \mathrm{C} / \mathrm{min}$. Lyophilized liposomes were equilibrated once the temperature exceeded the gel-to-liquid crystal temperature, leading to disruption of their original state soon after freeze-drying ${ }^{25)}$. Thus, the first heating process was adopted as data for lyophilized liposomes. All measurements were conducted under flowing nitrogen gas $(30 \mathrm{~mL} / \mathrm{min})$ through a furnace, and empty pans were used as reference.

\section{Results and Discussion}

\subsection{Structural integrity of DPPC liposomes after FD/RH}

If DPPC liposomes were freeze-dried without lyoprotectants, the liposomes aggregated and/or fused, which resulted in increment of the liposome sizes. To investigate whether liposomes aggregate and/or fuse during the $\mathrm{FD} /$ $\mathrm{RH}$ processes, we compare the sizes of the hydrated liposomes before and after the FD/RH processes (Fig. 2). Before the FD/RH processes, the mean size of the hydrated liposomes was 14-169 nm and there is no clear dependency of the alkyl chain length of $\mathrm{SB}_{n}$ on the liposome sizes. After FD/RH processes, the size of the hydrated liposomes that are prepared without $\mathrm{SB}_{n}$ increases from several tens of nanometers to several micrometers. This result indicates that the liposomes aggregate/fuse in the absence of $\mathrm{SB}_{n}$ during the $\mathrm{FD} / \mathrm{RH}$ processes. In contrast, when adding the sulfobetaines with an alkyl chain of intermediate length, $\mathrm{SB}_{n=6,8,10}$, the increment in the liposome size is remarkably prevented and the size distribution remains almost constant even after the $\mathrm{FD} / \mathrm{RH}$ processes. Addition of trehalose, which is known as a typical lyoprotectant, the size distribution of the liposomes remains almost constant after FD/RH. In the presence of alkyl sulfobetaines having shorter $\left(\mathrm{SB}_{n=1,2,4}\right)$ or longer $\left(\mathrm{SB}_{n=12,14,16,18}\right)$ alkyl chain lengths, the size of the liposomes increases after the FD/ $\mathrm{RH}$ processes. These results indicate that the length of the alkyl chain in $\mathrm{SB}_{n}$ is an important factor for lyoprotection of the DPPC liposomes. In our preliminary experiments, 


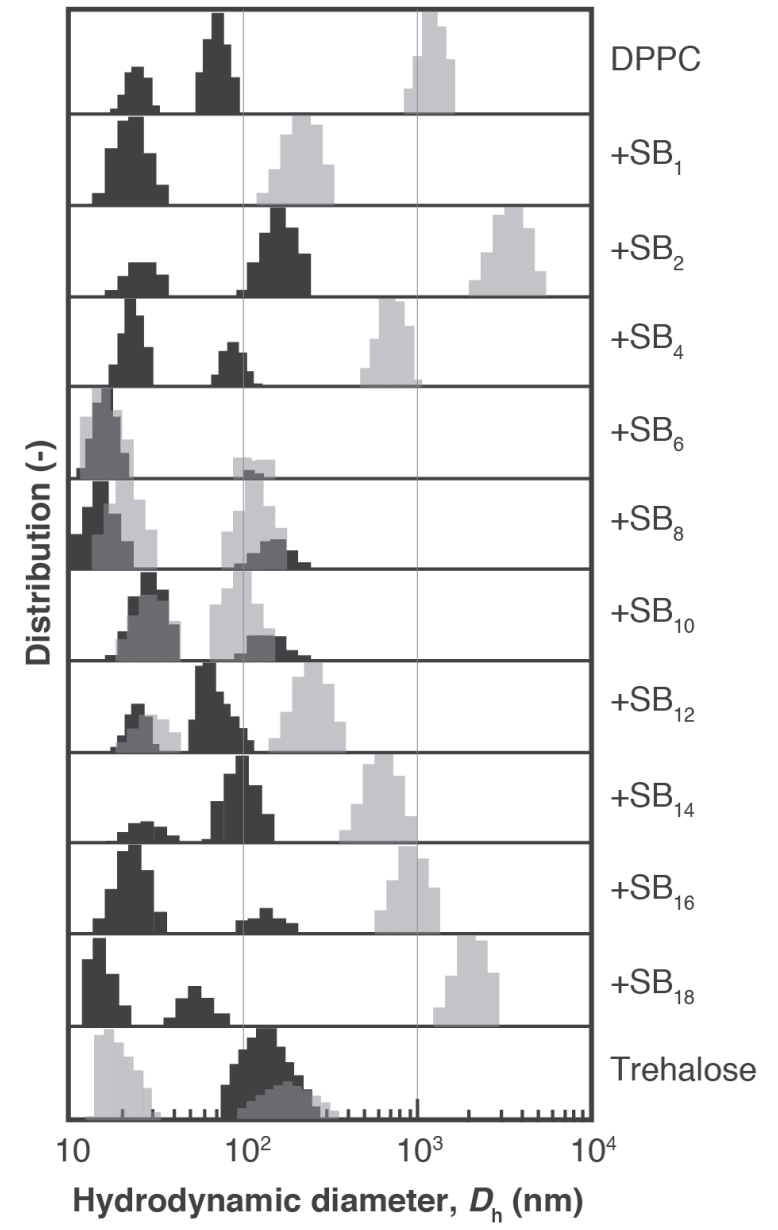

Fig. 2 Size distributions of the DPPC liposomes before (black bars) and after (gray bars) FD/RH. $\mathrm{SB}_{n}$ were added at $20 \mathrm{~mol} \%$ with respect to DPPC.

when using either a cationic or an anionic additive (20 mol\% with respect to DPPC, the same amount as the case of $\mathrm{SB}_{n}$ ), the DPPC liposomes aggregated and/or fused through the $\mathrm{FD} / \mathrm{RH}$ processes. In this experiment, alkyltrimethylammonium bromides or sodium alkyl sulfonates were used as cationic or anionic additives, respectively, with the same alkyl chain length as that of $\mathrm{SB}_{n}$ used above. Regardless of the alkyl chain length of these additives, aggregation and/or fusion of the liposomes was observed. These results indicate that the zwitterionic character and proper hydrophobicity (length of alkyl chain) of sulfobetaines may play an important role in prevention of aggregation and/or fusion of liposomes after the FD/RH processes.

For FF-TEM and the solute retention experiments in this study, $\mathrm{SB}_{n=10}$ was used as a representative additive. The FF-TEM observations reveal that the use of $\mathrm{SB}_{n=10}$ could prevent aggregation and/or fusion of the liposomes after $\mathrm{FD} / \mathrm{RH}$ (Fig. 3b). In the absence of additives, the liposomes aggregate and/or fuse after FD/RH(Fig. 3a). The results of FF-TEM are consistent with the results of DLS.

\section{Before FD/RH}

(a)

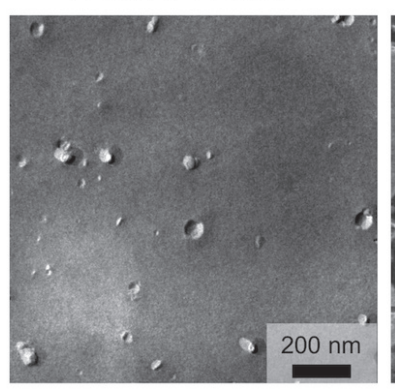

(b)

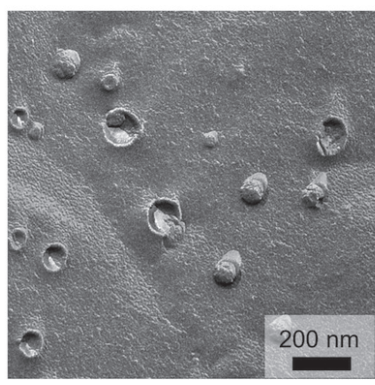

After FD/RH
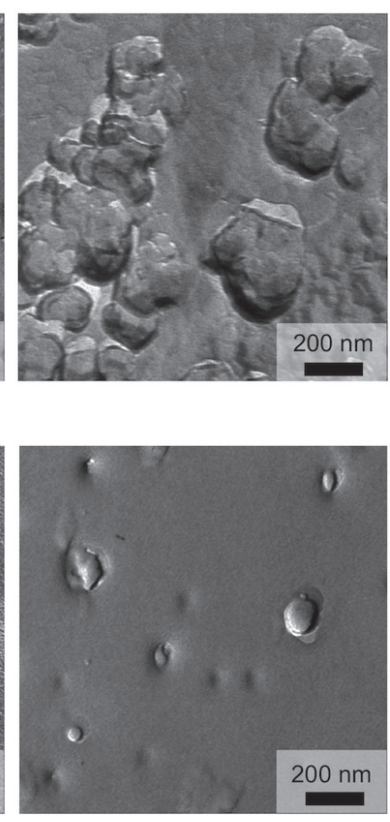

Fig. 3 FF-TEM images of liposomes before and after the $\mathrm{FD} / \mathrm{RH}$ processes. The images in (a) represent liposomes consisting of a single component of DPPC. The images in (b) represent liposomes containing DPPC and $20 \mathrm{~mol} \%$ of $\mathrm{SB}_{n=10}$ with respect to DPPC.

Table 1 Comparison of $\mathrm{CF}$ retention values of the liposomes.

\begin{tabular}{cc}
\hline Liposome formulation & CF retention (\%) \\
\hline DPPC & $<1$ \\
$\mathrm{DPPC}+\mathrm{SB}_{n=10}$ & $95 \pm 10$ \\
$\mathrm{DPPC}+$ Trehalose & $82 \pm 13$ \\
\hline
\end{tabular}

All values are presented as mean \pm standard deviation $(n=3)$.

Leakage of solutes entrapped in liposomes is caused by disruption of the lipid bilayer of the liposomes. Therefore, the amount of solutes entrapped in the liposomes after FD/ $\mathrm{RH}$ is a good indicator for investigating the integrity of the lipid bilayer during the $\mathrm{FD} / \mathrm{RH}$ processes. The results of $\mathrm{CF}$ retention values are summarized in Table 1 . CF retention values are $95 \pm 10 \%$ and $82 \pm 13 \%$ in the presence of $\mathrm{SB}_{n=10}$ or trehalose, respectively, significantly higher than those found in the absence of additives $(<1 \%)$. CF retention in the presence of $\mathrm{SB}_{n=10}$ is slightly but significantly larger compared with that in the presence of trehalose, an additive known to exert a significant lyoprotective effect ${ }^{20,21)}$. This result clearly shows that $\mathrm{SB}_{n=10}$ also provides significant lyoprotection for DPPC liposomes. Although use of either $\mathrm{SB}_{n=6}$ or $\mathrm{SB}_{n=8}$ could prevent disruption of the liposome membrane to some extent $(\mathrm{CF}$ retention $=57 \%$ for $\mathrm{SB}_{n=6}, 75 \%$ for $\mathrm{SB}_{n=8}$ ), its efficacy was lower than that of 
$\mathrm{SB}_{n=10}$.

\subsection{Effect of $\mathrm{SB}_{n}$ on the phase transitions of hydrated DPPC liposomes}

In general, the gel-to-liquid crystal phase transition temperature $\left(T_{\mathrm{m}}\right)$ of the phospholipid bilayer is strongly related to the intermolecular interaction between the lipid molecules constituting the lipid bilayer ${ }^{25,26)}$. For instance, if coexisting molecules (e.g. solvent $\left(\mathrm{H}_{2} \mathrm{O}\right)$, solute, additives) intercalate between lipids in the bilayer, the $T_{\mathrm{m}}$ of the lipid bilayer sometimes decreases. This decrease in the $T_{\mathrm{m}}$ is generally explained by reduction in intermolecular interaction between the lipids in the bilayer, which is caused by an increase in spacing between the lipids ${ }^{27}$. The $T_{\mathrm{m}}$ of hy-

(a)

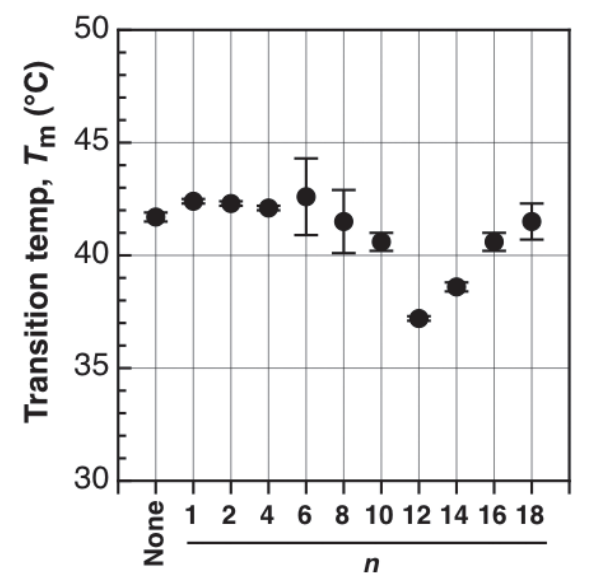

(b)

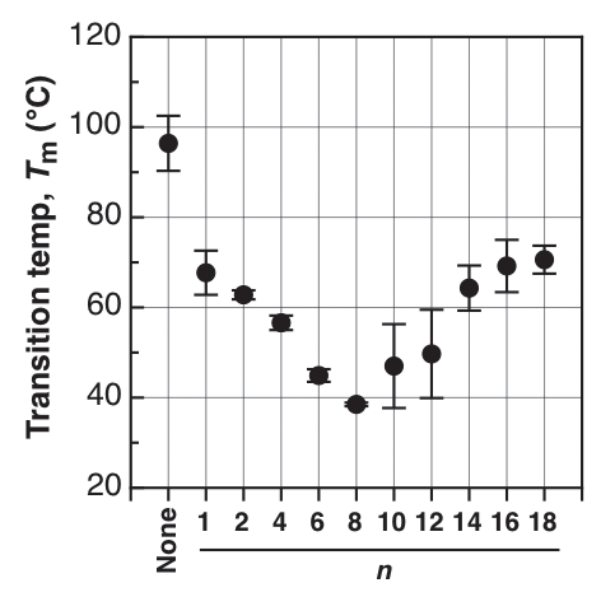

Fig. 4 Plots of gel-to-liquid crystal phase transition temperatures $\left(T_{\mathrm{m}}\right)$ for the hydrated (a) and freezedried (b) DPPC liposomes prepared in the presence of $20 \mathrm{~mol} \% \mathrm{SB}_{n}$ with respect to DPPC. Data for the hydrated and freeze-dried liposomes were acquired at 2 nd or 1 st heating process, respectively $\left(2^{\circ} \mathrm{C} /\right.$ min). Data are represented as mean \pm standard deviation $(n \geq 2)$. drated liposomes composed of pure DPPC was $41.7 \pm 0.2^{\circ} \mathrm{C}$ (Fig. 4a), which is consistent with general value reported previously ${ }^{25)}$. The $T_{\mathrm{m}}$ of the hydrated liposomes shifted in the presence of $\mathrm{SB}_{n}$, indicating that the interaction between DPPC in the hydrated lipid bilayer was altered by the addition of $\mathrm{SB}_{n}$.

The addition of alkyl sulfobetaines having a shorter alkyl chain, $\mathrm{SB}_{n=1,2,4,6}$, results in a slight increase in $T_{\mathrm{m}}$ of the hydrated liposomes $\left(\Delta T_{\mathrm{m}}=+0.4\right.$ to $\left.+0.9^{\circ} \mathrm{C}\right)$. This increase in the $T_{\mathrm{m}}$ can be explained by a kosmotropic effect, which can be observed when adding saccharides to liposomes ${ }^{28)}$. Kosmotropes are molecules that can stabilize the structure of water molecules in an aqueous solution. It has been known that kosmotropes, such as saccharides, reduce the amount of water molecules on the surface of lipid bilayers owing to local osmotic imbalance ${ }^{29)}$. This partial dehydration at the lipid bilayer surface should increase the $T_{\mathrm{m}}$ by decreasing the spacing between the lipids, which enhances intermolecular interaction between the lipids. At the same time, the amount of $\mathrm{SB}_{n=1,2,4,6}$ partitioned in the lipid bilayer should be quite low because of their relatively short alkyl chain. Most of the $\mathrm{SB}_{n=1,2,4,6}$ may be existed in the bulk solution.

As shown in Fig. 4a, in the presence of $\mathrm{SB}_{n=8,10,12,14,16,18}$, the $T_{m}$ of the aqueous dispersion decreases $\left(\Delta T_{\mathrm{m}}=-4.5\right.$ to $-0.2^{\circ} \mathrm{C}$ ). The kosmotropic effect of alkyl sulfobetaines should be overshadowed by the hydrophobic interaction between their alkyl chains in the $\mathrm{SB}_{n=8,10,12,14,16,18}$ and the palmitoyl groups in DPPC in the lipid bilayer. The amount of the $\mathrm{SB}_{n=8,10,12,14,16,18}$ that partitioned in the lipid bilayer should be much higher than that of the $\mathrm{SB}_{n=1-6}$ because of their relatively long alkyl chain. Within this range of alkyl chain length, a descending trend in the $T_{\mathrm{m}}$ is observed until $n$ value reaches $12 . \mathrm{SB}_{n=8,10,12}$ intercalated in the lipid bilayer should create "voids" in the hydrophobic region in the lipid bilayer because the alkyl chains of $\mathrm{SB}_{n=8,10,12}$ are shorter than the acyl chains of DPPC. These "voids" can contribute to the reduction in the $T_{\mathrm{m}}$ by reducing intermolecular interaction between the lipids in the bilayer, which is also discussed in the next section regarding the freezedried bilayers. When using $\mathrm{SB}_{n=14,16,18}$, there is an increasing trend in the $T_{\mathrm{m}}$. The hydrophobic interaction should contribute to an increase in the intermolecular interaction between the lipids in the bilayer. It can be assumed that these longer alkyl chains in $\mathrm{SB}_{n=14,16,18}$ should fill the "voids" in the hydrophobic region in the lipid bilayer, which contribute to the recovery of the intermolecular interaction between the lipids in the lipid bilayer.

\subsection{Effect of $\mathrm{SB}_{n}$ on the phase transitions of freeze-dried DPPC liposomes}

It is important to determine the $T_{\mathrm{m}}$ to clarify the extent of intercalation of additives in freeze-dried lipid bilayers. The DSC thermograms for freeze-dried liposomes were 


\section{T. Aikawa, K. Sato, H. Okado et al.}

shown in Fig. S4(b). In the absence of $\mathrm{SB}_{n}, T_{\mathrm{m}}$ of the freeze-dried liposome is $96.4 \pm 6.1^{\circ} \mathrm{C}$ (Fig. 4b), which is consistent with previously published data ${ }^{26)}$. In contrast, in the presence of $\mathrm{SB}_{n}$ regardless of their alkyl chain length, the $T_{\mathrm{m}}$ of the freeze-dried liposomes is lower compared to that in the absence of additives. This result can be explained by the intercalation of $\mathrm{SB}_{n}$ into the freeze-dried lipid bilayer. The trend of reduction in $T_{\mathrm{m}}$ for the freezedried liposomes is different from those of the hydrated liposomes. For instance, even when using alkyl sulfobetaines with shorter alkyl chain $\left(\mathrm{SB}_{n=1,2,4,6}\right)$, which increases the $T_{\mathrm{m}}$ of the hydrated liposomes (Fig. 4a), the $T_{\mathrm{m}}$ of the freezedried liposomes decreases compared to that without additives. When using $\mathrm{SB}_{n=1,2,4,6,8}$, the $T_{\mathrm{m}}$ of the freeze-dried liposomes decreases with increasing $n$-value. When using $\mathrm{SB}_{n=6,8,10}$, the $T_{\mathrm{m}}$ of the freeze-dried liposomes remarkably decreases. Particularly, when using $\mathrm{SB}_{n=8}$, the $T_{\mathrm{m}}$ of the freeze-dried liposomes reaches the lowest value(38.5 \pm $0.4^{\circ} \mathrm{C}$ ), which is lower than that of the fully hydrated pure DPPC liposomes $\left(41.7 \pm 0.2^{\circ} \mathrm{C}\right)$. Moreover, multiple endothermic peaks were observed when using $\mathrm{SB}_{n=6,8,10,12}$ (Fig. S4(b)), which indicates that phase state of the freezedried lipid may be different from that without $\mathrm{SB}_{n}$. The powder x-ray diffraction revealed that crystallinity of freeze-dried DPPC liposomes containing $\mathrm{SB}_{n=10}$ was the same with that composed pure DPPC (data not shown). Further examination should be required to reveal details in packing of lipids in the freeze-dried liposome in the presence of $\mathrm{SB}_{n}$.

The interaction between $\mathrm{SB}_{n}$ and the freeze-dried lipid bilayer can be illustrated by considering the trend of the $T_{\mathrm{m}}$ reduction compared with the alkyl chain length of $\mathrm{SB}_{n}$ (Scheme 1). $\mathrm{SB}_{n}$ with shorter alkyl chain length could create larger "void" spaces in the freeze-dried bilayers owing to their much shorter alkyl chain length compared to the palmitoyl groups in DPPC (Scheme 1, left panel). However, the amount of $\mathrm{SB}_{n}$ intercalated in the bilayer should be lower because of the low hydrophobicity of the shorter alkyl chain. Thus, the total space of "voids" in the bilayers reduced, which did not strongly influence the reduction of the $T_{\mathrm{m}}$ interaction between $\mathrm{SB}_{n=1}$ and phosphocholines of DPPC.

$\mathrm{SB}_{n}$ having alkyl chain of intermediate length should create the largest total space of "voids" in the freeze-dried bilayers (Scheme 1, middle panel), which results in the marked reduction of the $T_{\mathrm{m}}$. The largest amount of total "void" space should arise from the increased amount of intercalated $\mathrm{SB}_{n}$ in the bilayers and their shorter alkyl chain compared to the palmitoyl groups in the DPPC, which can still create "voids" in the DPPC bilayer.

The use of $\mathrm{SB}_{n}$ with longer alkyl chain led to an increase in the $T_{\mathrm{m}}$ of the freeze-dried liposomes, indicating that the intermolecular interaction of the freeze-dried bilayer is recovered by filling "voids" owing to their longer alkyl chains (Scheme 1, right panel). The amount of $\mathrm{SB}_{n}$ intercalated in the bilayer further increased due to adequate hydrophobicity of their longer alkyl chain.

The changes in $T_{\mathrm{m}}$ that were observed when using trehalose, which is one of the most effective lyoprotectants ${ }^{21,22}$, were similar to those observed with $\mathrm{SB}_{n}$. It has been reported that the addition of trehalose decreases the $T_{\mathrm{m}}$ of the freeze-dried liposomes $\left(\sim 25^{\circ} \mathrm{C}\right.$ for dried DPPC liposomes $^{26)}$ ). The lyoprotection mechanism of liposome using trehalose has been explained by the water replacement model $^{30,31)}$. During the FD process, trehalose can intercalate between lipids in the dried bilayer via hydrogen bonding between headgroups of lipids and hydroxyl groups in trehalose. This resulted in a marked reduction in the $T_{\mathrm{m}}$ of the freeze-dried liposomes. Thus, reduction in the $T_{\mathrm{m}}$ of freeze-dried liposomes by addition of $\mathrm{SB}_{n}$ implies a possi-

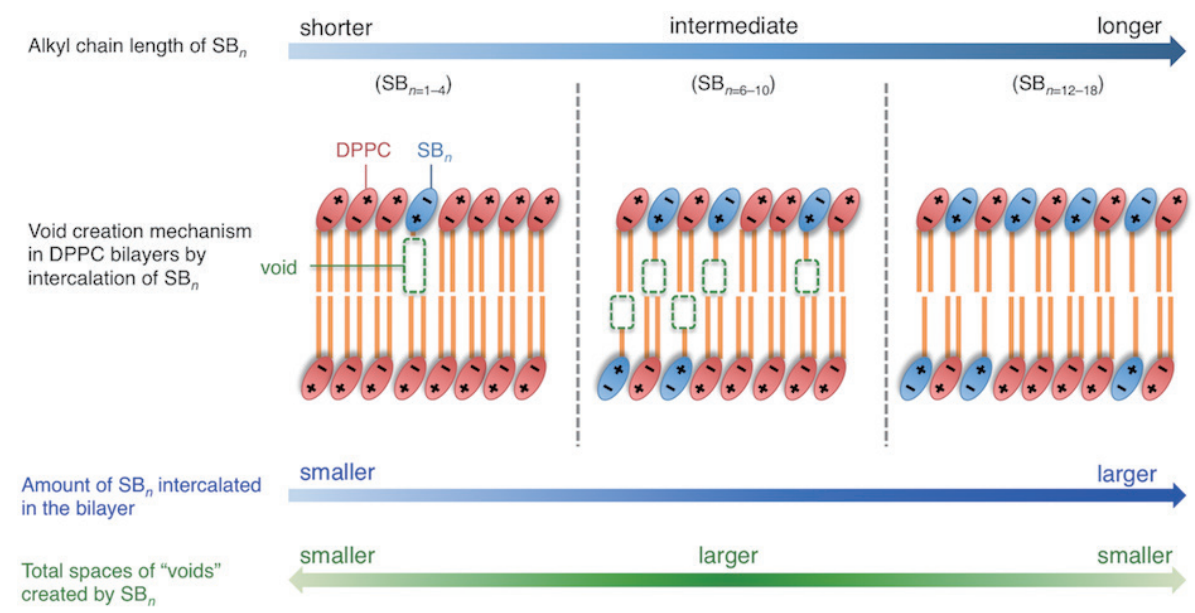

Scheme 1 Schematic of "void" creation mechanism in freeze-dried bilayers by intercalation of $\mathrm{SB}_{n}$. The total "voids" space in the bilayers should be dependent on the amount of intercalated $\mathrm{SB}_{n}$ and the alkyl chain length of the intercalated $\mathrm{SB}_{n}$. 
bility for replacement of water molecules bound to lipid headgroups with $\mathrm{SB}_{n}$ during the FD process. Although water molecules bound to lipid headgroups may be replaced with $\mathrm{SB}_{n}$, there should be a difference in the underlying mechanism responsible for the $T_{\mathrm{m}}$ reduction between trehalose and $\mathrm{SB}_{n}$. In the case of $\mathrm{SB}_{n}$, the $T_{\mathrm{m}}$ reduction of the freeze-dried liposomes should be related to the "void" created in the hydrophobic region in the bilayer, which is dominated by the hydrophobic interaction between short alkyl chains of the intercalated $\mathrm{SB}_{n}$ and palmitoyl groups in the DPPC (Scheme 1, middle panel). In contrast, in the case of trehalose, hydrogen bonding between the hydroxyl groups in the trehalose and the lipid headgroups may be involved in the $T_{\mathrm{m}}$ reduction of freeze-dried liposomes.

It has been reported that liposome disruption is frequently induced during the gel-to-liquid crystal phase transition during the $\mathrm{RH}$ process ${ }^{32)}$. Therefore, limiting the phase transition during the $\mathrm{RH}$ process should be important in preventing liposome disruption. It has been shown that when the $T_{\mathrm{m}}$ of the freeze-dried liposomes is lower than the temperature of the $\mathrm{RH}$ process, the gel-to-liquid crystal phase transition of the liposomes can be prevented during the $\mathrm{RH}$ process. Thus, it can be assumed that the lyoprotective effect in the present study is related to the markedly reduced $T_{\mathrm{m}}$ of the freeze-dried liposomes. Taken together, molecules that are good candidates for lyoprotectants of lipid bilayers should not solubilize the bilayer in the hydrated state, and should strongly interact with the lipid headgroups in the dried state, leading to the $T_{\mathrm{m}}$ reduction of the dried bilayer.

\section{Conclusions}

The present study demonstrated lyoprotection of DPPC liposomes by using $\mathrm{SB}_{n}$. The alkyl chain length of $\mathrm{SB}_{n}$ was a crucial factor for the lyoprotective effect. $\mathrm{SB}_{n}$ having an alkyl chain with intermediate length exerted lyoprotective effect. The lyoprotection offered by $\mathrm{SB}_{n}$ may be explained by its prevention of the gel-to-liquid crystal phase transition during the rehydration of freeze-dried liposomes, which can be achieved by a reduction of the phase transition temperature of the freeze-dried liposomes, to a temperature lower than that of the rehydration process. The use of $\mathrm{SB}_{n}$ having an alkyl chain with intermediate length should increase the total "void" space in the hydrocarbon region in the freeze-dried bilayer owing to their appropriate hydrophobicity and shorter alkyl chain compared to the acyl groups of DPPC. The results from this study provide an insight into the mechanism of zwitterionic amphiphiles for preserving lipid bilayers during freeze-drying.

\section{Acknowledgements}

This work was supported by the Oil \& Fat Industry Kaikan, the Terumo Foundation for Life Sciences and Arts, and JSPS KAKENHI Grant Number JP16K21398(T.A.) .

\section{Supporting Information}

Synthesis of alkyl sulfobetaines used in this study and the DSC thermograms.

This material is available free of charge via the Internet at http://dx.doi.org/jos.66.10.5650/jos.ess. 17100

\section{References}

1) Kraft, J.; Freeling, J.; Wang, Z.; Ho, R.; Emerging research and clinical development trends of liposome and lipid nanoparticle drug delivery systems. $J$. Pharm. Sci. 103, 29-52 (2014).

2) Allen, T.; Cullis, P.; Liposomal drug delivery systems: From concept to clinical applications. Adv. Drug Delivery Rev. 65, 36-48 (2013).

3) Furuki, T.; Watanabe, T.; Furuta, T.; Takano, K.; Shirakashi, R.; Sakurai, M. The dry preservation of giant vesicles using a group 3 LEA protein model peptide and its molecular mechanism. Bull. Chem. Soc. Jpn. 89, 1493-1499 (2016).

4) Nawa, E.; Yamamoto, D.; Shioi, A. Chemotactic amoeboid-like shape change of a vesicle under a $\mathrm{pH}$ gradient. Bull. Chem. Soc. Jpn. 88, 1536-1544(2015).

5) Liu, X.; Situ, A.; Kang, Y.; Villabroza, K.; Liao, Y.; Chang, C.; Donahue, T.; Nel, A.; Meng, H. Irinotecan delivery by lipid-coated mesoporous silica nanoparticles shows improved efficacy and safety over liposomes for pancreatic cancer. ACS Nano 10, 2702-2715 (2016).

6) Luo, D.; Carter, K.; Razi, A.; Geng, J.; Shao, S.; Giraldo, D.; Sunar, U.; Ortega, J.; Lovell, J. Doxorubicin encapsulated in stealth liposomes conferred with light-triggered drug release. Biomaterials 75, 193-202 (2016).

7) Dai, Y-Q.; Qin G.; Geng, S-Y.; Yang, B.; Xu, Q.; Wang, $\mathrm{J}$-Y.; Photo-responsive release of ascorbic acid and catalase in CDBA-liposome for commercial application as a sunscreen cosmetic. RSC Adv. 2, 3340-3346 (2012).

8) Betz, G.; Aeppli, A.; Menshutina, N.; Leuenberger, H. In vivo comparison of various liposome formulations for cosmetic application. Int. J. Pharm. 296, 44-54 (2005).

9) Malheiros, P.; Daroit, D.; Brandelli, A.; Food applications of liposome-encapsulated antimicrobial peptides. Trends Food Sci. Technol. 21, 284-292 (2010).

10） Mozafari, M.R.; Johnson, C.; Hatziantoniou, S.; Demet- 


\section{T. Aikawa, K. Sato, H. Okado et al.}

zos, C. Nanoliposomes and their applications in food nanotechnology. J. Liposome Res. 18, 309-327 (2008).

11) Taylor, T.M.; Weiss, J.; Davidson, P.M.; Bruce, B.D. Liposomal nanocapsules in food science and agriculture. Crit. Rev. Food Sci. Nutr. 45, 587-605(2005).

12) Grit, M.; Crommelin, D. Chemical stability of liposomes: implications for their physical stability. Chem. Phys. Lipids 64, 3-18(1993).

13) Frankel, E.N. Lipid oxidation: Mechanisms, products and biological significance. J. Am. Oil Chem. Soc. 61, 1908-1917(1984).

14) Chatterjee, S.N.; Agarwal, S. Liposomes as membrane model for study of lipid peroxidation. Free Radical Biol. Med. 4, 51-72 (1988).

15) Chen, C.; Han, D.; Cai, C.; Tang, X. An overview of liposome lyophilization and its future potential. J. Control. Release 142, 299-311(2010).

16) Mohammed, A.R.; Coombes, A.G.A.; Perrie, Y. Amino acids as cryoprotectants for liposomal delivery systems. Eur. J. Pharm. Sci. 30, 406-413(2007).

17) Withers, L.A.; King, P.J. Proline: A novel cryoprotectant for the freeze preservation of cultured cells of Zea mays L. Plant Physiol. 64, 675-678(1979).

18) Crowe, L.M.; Womersley, C.; Crowe, J.H.; Reid, D.; Appel, L.; Rudolph, A. Prevention of fusion and leakage in freeze-dried liposomes by carbohydrates. Biochim. Biophys. Acta 861, 131-140(1986).

19) Crowe, J.H.; Oliver, A.E.; Hoekstra, F.A.; Crowe, L.M. Stabilization of dry membranes by mixtures of hydroxyethyl starch and glucose: The role of vitrification. Cryobiology 35, 20-30 (1997).

20) Crowe, J.H.; Crowe, L.M.; Chapman, D. Preservation of membranes in anhydrobiotic organisms: The role of trehalose. Science 223, 701-703 (1984).

21) Crowe, L.M.; Crowe, J.H.; Rudolph, A.; Womersley, C.; Appel, L. Preservation of freeze-dried liposomes by trehalose. Arch. Biochem. Biophys. 242, 240-247 (1985)

22) Oliver, A.E.; Hincha, D.K.; Tsvetkova, N.M.; Vigh, L.; Crowe, J.H. The effect of arbutin on membrane integrity during drying is mediated by stabilization of the lamellar phase in the presence of nonbilayer-forming lipids. Chem. Phys. Lipids, 111, 37-57(2001).

23) Williams, W.P.; Quinn, P.J.; Tsonev, L.I.; Koynova, R.D. The effects of glycerol on the phase behaviour of hydrated distearoylphosphatidylethanolamine and its possible relation to the mode of action of cryoprotectants. Biochim. Biophys. Acta 1062, 123-132(1991).

24) Aikawa, T.; Yokota, K.; Kondo, T.; Yuasa, M. Intermolecular interaction between phosphatidylcholine and sulfobetaine lipid: A combination of lipids with antiparallel arranged headgroup charge. Langmuir 32, 10483-10490 (2016).

25) Ohtake, S.; Schebor, C.; de Pablo, J.J. Effects of trehalose on the phase behavior of DPPC-cholesterol unilamellar vesicles. Biochim. Biophys. Acta 1758, 65-73 (2006).

26) Ohtake, S.; Schebor, C.; Palecek, S.P.; de Pablo, J.J. Phase behavior of freeze-dried phospholipid-cholesterol mixtures stabilized with trehalose. Biochim. Biophys. Acta 1713, 57-64(2005).

27) Crowe, J.H.; Carpenter, J.F.; Crowe, L.M. The role of vitrification in anhydrobiosis. Annu. Rev. Physiol. 60, 73-103 (1998).

28) Crowe, L.M.; Crowe, J.H. Solution effects on the thermotropic phase transition of unilamellar liposomes. Biochim. Biophys. Acta 1064, 267-274(1991).

29) Andersen, H.D.; Wang, C.; Arleth, L.; Peters, G.H.; Westh, P. Reconciliation of opposing views on membrane-sugar interactions. Proc. Natl. Acad. Sci. USA 108, 1874-1878(2011).

30) Crowe J.H.; Hoekstra, F.A.; Nguyen, K.H.; Crowe, L.M. Is vitrification involved in depression of the phase transition temperature in dry phospholipids? Biochim. Biophys. Acta 1280, 187-196(1996).

31) Crowe, L.M.; Crowe. J.H. Trehalose and dry dipalmitoylphosphatidylcholine revisited. Biochim. Biophys. Acta 946, 193-201 (1988).

32) Hays, L.M.; Crowe, J.H.; Wolkers, W.; Rudenko, S. Factors affecting leakage of trapped solutes from phospholipid vesicles during thermotropic phase transitions. Cryobiology 42, 88-102(2001). 